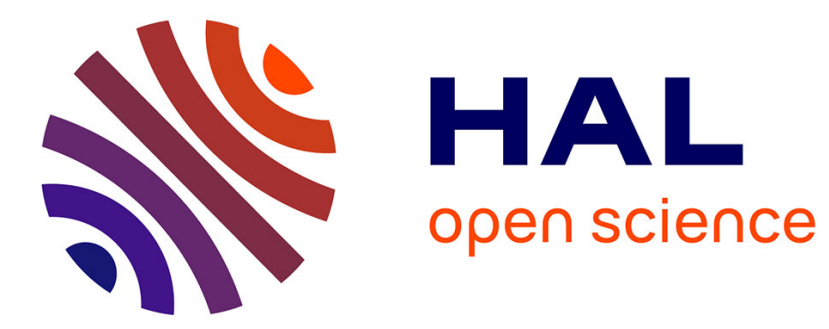

\title{
La voix de l'expulsé
}

Clara Lecadet

\section{- To cite this version:}

Clara Lecadet. La voix de l'expulsé. Plein Droit, 2015, Les expulsés : leurs voix, leurs droits, 107 (4), pp.7-10. 10.3917/pld.107.0007 . hal-01809492

\section{HAL Id: hal-01809492 \\ https://hal.science/hal-01809492}

Submitted on 6 Jun 2018

HAL is a multi-disciplinary open access archive for the deposit and dissemination of scientific research documents, whether they are published or not. The documents may come from teaching and research institutions in France or abroad, or from public or private research centers.
L'archive ouverte pluridisciplinaire HAL, est destinée au dépôt et à la diffusion de documents scientifiques de niveau recherche, publiés ou non, émanant des établissements d'enseignement et de recherche français ou étrangers, des laboratoires publics ou privés. 


\section{LA VOIX DE L'EXPULSÉ}

\section{Clara Lecadet}

\section{GISTI | « Plein droit »}

2015/4 n 107 | pages 7 à 10

ISSN 0987-3260

Article disponible en ligne à l'adresse :

http://www.cairn.info/revue-plein-droit-2015-4-page-7.htm

\section{!Pour citer cet article :}

Clara Lecadet, « La voix de l'expulsé », Plein droit 2015/4 (n 107), p. 7-10.

Distribution électronique Cairn.info pour GISTI.

(C) GISTI. Tous droits réservés pour tous pays.

La reproduction ou représentation de cet article, notamment par photocopie, n'est autorisée que dans les limites des conditions générales d'utilisation du site ou, le cas échéant, des conditions générales de la licence souscrite par votre établissement. Toute autre reproduction ou représentation, en tout ou partie, sous quelque forme et de quelque manière que ce soit, est interdite sauf accord préalable et écrit de l'éditeur, en dehors des cas prévus par la législation en vigueur en France. Il est précisé que son stockage dans une base de données est également interdit. 
L'émergence des expulsés dans l'espace politique de leur pays d'origine fait écho aux questions portées par les sans-papiers en France. Elle s'explique par les conditions mêmes de l'expulsion et par la situation d'abandon des expulsés à leur retour faute de soutien institutionnel. Encore faut-il que le contexte politique soit propice, que les expulsés ne soient pas baillonnés. Ou que leur expression ne soit pas mise en sourdine par une institutionnalisation qui fait le jeu des pays qui expulsent.

\section{La voix de l'expulsé}

Clara Lecadet, anthropologue, chercheure associée à l'Institut interdisciplinaire de l'anthropologie du contemporain-Laboratoire d'anthropologie urbanités mondialisations, EHESS-CNRS

En août 1996, au moment de l'évacuation de l'église SaintBernard, c'est déjà sur une autre scène que se jouent le sort du mouvement des sans-papiers et l'échec des négociations autour d'une régularisation globale. À Bamako, les expulsés maliens de Saint-Bernard retrouvent d'autres compatriotes expulsés d'Angola, de Zambie, d'Arabie Saoudite, de Tanzanie. Les pertes liées à l'expulsion mais aussi le sentiment de leur inexistence politique après plusieurs appels vains à leur gouvernement, forment entre eux une communauté d'expérience. Sous l'égide
d'Ousmane Diarra, un commerçant malien expulsé d'Angola, ils créent en 1996 l'Association malienne des expulsés (AME), une initiative pionnière. La voix de l'expulsé, un manifeste daté de $1997^{1}$, en fixe les enjeux. Ils font écho aux questions portées par le mouvement des sanspapiers en France, qui a trouvé à se rassembler autour d'un nom et de l'autonomie des luttes ${ }^{2}$.

L'émergence des expulsés dans l'espace public est liée à un processus de prise de parole sur les expulsions, dans leur double statut d'expérience et de question politique. L'incapacité de l'État malien à protéger ses ressortissants est présentée comme la cause majeure du caractère unilatéral des expulsions, des droits bafoués et des biens confisqués. Le texte dénonce l'opacité des négociations entre les gouvernements malien et angolais au sujet des biens confisqués des Maliens expulsés d'Angola, et accuse l'État malien de faciliter l'expulsion des Maliens depuis la France, en monnayant la délivrance par son consulat des laissez-passer nécessaires à la mise en œuvre d'une expulsion. Il se termine par un appel, qui est à la fois une injonction à l'État malien et au soulèvement populaire: "L'Association malienne des expulsés sollicite aujourd'bui la force vive et décisive malienne dans cette lutte afin que les biens confisqués en Angola, en France ou ailleurs soient immédiatement restitués sans conditions; que les expulsions soient immédiatement arrêtées, que les autorités diplomatiques maliennes en France soient remplacées, que les expulsés se retournent dans leurs pays hôtes, que des mesures sécurisantes soient prises pour protéger les personnes et leurs biens. » S'en est 
suivi un mouvement de contestation des politiques d'expulsion qui s'est développé au Mali mais s'est également diffusé dans d'autres États africains au cours des années 2000, ainsi qu'un essaim d'initiatives qui ont contribué à hisser les expulsés au rang d'acteurs sur les questions touchant à l'accueil et aux droits des expulsés.

\section{Initiatives reproductibles}

L'émergence et la diffusion d'un mouvement propre aux expulsés en Afrique ont d'abord une explication conjoncturelle; ce sont dans les conditions de l'expulsion et l'abandon dans lequel elle place les migrants, qu'il faut chercher la genèse de leur organisation collective. Le lien génétique entre les conditions, les lieux de l'expulsion, et l'émergence de mobilisations, permet de comprendre le processus de politisation des expulsés. Il s'agit de créer un espace de revendication mais aussi, et peutêtre surtout, de donner un lieu temporaire à ceux qui reviennent démunis et sans attaches. À partir de 2006, l'AME met ainsi en place un dispositif d'accueil à l'aéroport de Bamako-Sénou et d'hébergement des expulsés dans les locaux de l'association. Une grande partie des associations créées par les expulsés au Mali dans le sillage de l'AME, est mue par cette nécessité de trouver des formes d'accueil et d'hébergement d'urgence au niveau de l'aéroport (pour les expulsions par voie aérienne) et aux abords des frontières (pour les expulsions collectives par voie terrestre depuis la Libye, l'Algérie, la Mauritanie). La création en 2006 à Bamako de l'Association des refoulés d'Afrique centrale au Mali (Aracem), fait ainsi suite au parcours d'errance de Roméo N'Tamag et Patrice Boukar, deux Camerounais expulsés d'Algérie, soucieux de donner une visibilité à la situation particulière des étrangers originaires d'Afrique centrale expulsés sur le sol malien. Leur inscription entre 2009 et 2011 dans un programme financé par le Pnud, avec les ONG Medico International et Médecins du Monde, permet l'ouverture à Bamako d'une maison d'accueil pour les expulsés d'Algérie et de Libye. Ces lieux visent à pallier l'absence de soutien institutionnel dans la période post-expulsion. Mais la création d'associations par des expulsés est également venue formaliser des lieux déjà constitués. Au nord du Mali, à Kidal, c'est sur l'injonction du gouverneur que Modibo Diakité a créé en 2008 l'Association d'appui aux migrants refoulés de la commune urbaine de Kidal (AMRK), afin de donner à l'existence de son foyer, ouvert en 2003 pour accueillir les expulsés d'Algérie, une existence sociale. Ainsi, le processus associatif a, selon les cas, précédé ou simplement formalisé la mise en place de lieux d'accueil.

D'autres acteurs sont intervenus au cours des années 2000 sur la question de l'après-expulsion, un domaine jusque-là livré à l'autogestion et aux seules capacités d'organisation des expulsés. En 2006, Aminata Dramane Traoré, essayiste et ancienne ministre de la culture au Mali, accueille à l'aéroport de Bamako les jeunes rescapés maliens de la répression sanglante devant l'enclave espagnole de Ceuta et Melilla. Elle crée l'Association retour travail et dignité (ARTD), destinée à réinsérer ceux qu'elle nomme les «fils maudits » du pays. L'ARTD se dissout et se reforme quelques mois plus tard en dehors de la tutelle jugée pesante de sa fondatrice. De façon plus générale, la répression de Ceuta et Melilla, a provoqué une onde de choc et constitué un tournant dans la conscientisation des épreuves vécues par les migrants. Ces événements ont, dans certains cas, sorti ponctuellement les pouvoirs publics de leur attentisme vis-à-vis de l'expulsion de leurs ressortissants. En 2005, le gouvernement camerounais organise ainsi le rapatriement en trois vols des survivants camerounais de Ceuta et Melilla, suivi d'une cérémonie officielle et de la remise d'une somme d'argent. L'Association des rapatriés et de lutte contre l'émigration clandestine du Cameroun (ARECC) a été créée en 2005 à Yaoundé par Robert Alain Lipothy et des jeunes issus de ces rapatriements. En 2006, Oskar Eyezo'o, expulsé de France, crée l'association Welcome Back Cameroon. La création en 2008 par Razak Aboubacar de l'Association togolaise des expulsés (ATE), s'aligne explicitement sur le modèle de l'AME. Ces initiatives reproductibles s'engendrent les unes les autres : l'Union des jeunes rapatriés togolais voit le jour dans le sillage de l'ATE peu après.

S'engage alors un travail discret de mise en commun des savoirs et des expériences de lutte: en jeu, la recherche de partenariats avec des ONG du Nord, sources de financement, mais aussi l'apprentissage de la bureaucratie associative, ou encore l'harmonisation des discours et des registres d'action. Une délégation de l'AME rencontre les membres de l'ATE à Sokodé en 2010, un partage d'expériences poursuivi par la venue en 2014 de membres de l'ATE à Bamako. Ces échanges souffrent cependant de l'hétérogénéité des contextes nationaux.

Selon les pays, le déploiement des mobilisations est facilité ou au contraire empêché et ne peut être ramené à un schéma d'action collective simple. L'action pionnière de l'AME a, en effet, été favorisée par la libéralisation démocratique au Mali en 1991, qui a permis à 
des catégories marginalisées de la société d'apparaître dans l'espace public. La radio Kayira, proche du parti Sadi, dédie une large part de ses programmes à la voix des sansvoix, dont les expulsés deviennent une figure emblématique. En 1998, une marche contre l'emprisonnement de 77 Maliens expulsés par le $\ll 38^{\mathrm{e}}$ charter Debré » et leur libération deux semaines plus tard sont largement relayées dans les médias. Les expulsés commencent à témoigner publiquement de leur expérience et à dénoncer l'abandon de leur propre gouvernement. L'organisation à partir de 2007 de journées de débat public ainsi que la campagne de 2008 contre la signature d'« accords de réadmission » entre la France et le Mali, dénoncés par l'AME comme un blanc-seing donné à la France pour expulser et auxquels le gouvernement malien finit par renoncer, hissent l'AME au rang d'un véritable acteur politique. L'inscription de l'AME dans un réseau d'ONG et d'associations européennes engagées sur la question des droits des immigrés, donne un écho international à ces mobilisations, qui s'inscrivent dans le sillage du mouvement européen contre l'adoption de la directive « retour ».

\section{Ecoutés, arrêtés ou soumis}

Les mobilisations n'ont pas pris une ampleur similaire au Togo et au Cameroun. Ces États, ayant toujours fait discrètement allégeance à la souveraineté des États en matière de politique migratoire, n'ont jamais considéré, comme ce fut le cas au Mali depuis l'indépendance, leurs émigrés comme un apport essentiel à la construction nationale.

$\mathrm{Au}$ Togo, l'exil d'un très grand nombre d'opposants politiques à la suite de l'échec de la transition démocratique au début des années 1990, a rendu la diaspora suspecte aux yeux du régime. À leur retour, les expulsés, immédiatement catalogués par la police de l'aéroport de Lomé comme des ennemis du régime, étaient souvent emprisonnés jusqu'au tournant des années 2000, ou devaient négocier leur libération en jouant de leurs relations ou en remettant d'importantes sommes d'argent. L'ATE prend ainsi de nombreuses précautions sur les thèmes abordés dans les réunions publiques.

Plutôt que d'opter pour une confrontation directe avec le pouvoir politique à l'instar de l'AME, elle choisit des questions plus fédératrices, comme les formes légales de migration et l'apport de la diaspora dans l'économie togolaise. Elle s'inscrit dans un champ d'associations (Attac Togo, Visions Solidaires) qui, à Lomé, tentent d'établir des ponts entre le pouvoir politique et les demandes de la société civile, à l'origine, en 2010, du premier forum social togolais. En 2012, une des demandes exprimées par Razak Aboubacar, le président de l'ATE, lors d'une rencontre avec le ministre des affaires étrangères, porte sur la création d'un ministère des Togolais de l'extérieur, qui faciliterait l'obtention d'informations sur les expulsions depuis les pays de séjour des immigrés. Au Cameroun, les associations d'expulsés sont également freinées dans leur possibilité de mobilisation, dans un contexte néanmoins marqué par la montée en puissance du champ associatif et par la multiplication, au cours des années 2000, d'associations et d'ONG travaillant sur des thèmes inspirés par des programmes de financement européens et onusiens (migrations, citoyen- neté). Toutefois, comme au Sénégal, ces associations camerounaises ont davantage tendance à s'inscrire comme relais des messages portés par les autorités étatiques, par l'Europe et par l'Organisation internationale des migrations (OIM) sur le respect des formes légales de migration plutôt que de défendre des positions favorables au principe de la libre circulation. Ces positionnements variés face aux enjeux des politiques migratoires vont ainsi, parmi les associations d'expulsés, de l'opposition à l'alliance aux politiques étatiques.

Le mouvement des expulsés est par conséquent protéiforme et ne se résume pas à des mobilisations contre les États. Au Mali, en dépit du leadership de l'AME depuis les années 1990, toutes les associations n'ont pas adopté une position critique vis-à-vis des politiques étatiques. Le rapprochement d'une partie des associations d'expulsés créées au Mali avec des ONG européennes mais aussi avec l'OIM et le Centre d'information et de gestion des migrations (Cigem), projet pilote de l'Union européenne lancé en 2009 à Bamako pour une gestion concertée des migrations, les a transformées en prestataires des dispositifs d'aide au retour ${ }^{3}$ ainsi qu'en gestionnaires de l'assistance médicosociale aux expulsés. Atténuer les conséquences de l'expulsion par des mesures de type humanitaire a, de fait, désamorcé dans le discours de la plupart des associations chargées de leur mise en œuvre toute velléité de critique politique. De plus, la participation de certaines associations d'expulsés maliennes aux campagnes « d'information 
et de sensibilisation sur les risques de la migration irrégulière », organisées depuis 2008 par le ministère des Maliens de l'extérieur et de l'intégration africaine et par l'OIM, a parachevé ce processus d'instrumentalisation par les instances étatiques et les organismes en charge des migrations à l'échelle internationale. La posture radicale de l'association camerounaise ARECC rejoint également le discours de dissuasion porté par les autorités publiques et par l'OIM. Elle fait tourner en 2012, dans plusieurs villes camerounaises, une exposition de photos montrant les tragédies de la migration afin d'exhorter les jeunes à respecter les cadres de l'immigration légale. L'ARECC, qui se prévaut de la force du témoignage de ceux qui ont fait la route et en ont connu les épreuves, bénéficie d'une très rapide médiatisation ${ }^{4}$. Robert Alain Lipothy, son président, juge d'ailleurs sévèrement les mobilisations survenues au Mali et dans divers forums africains contre les expulsions, qu'il assimile à une vaine agitation.

L'auto-organisation des expulsés a ainsi pu devenir un relais des États dans leur injonction au respect des formes légales de la migration. L'AME a gardé dans ce processus d'enrôlement des associations d'expulsés au profit des intérêts politiques européens et onusiens en matière de réinsertion et de fixation des migrants expulsés, une voix critique et un positionnement politique sur la question des droits des migrants. Lors d'une caravane organisée en février 2011 pour rejoindre le forum social mondial à Dakar au Sénégal, elle est rejointe par l'ATE et Welcome Back Cameroon, ainsi que d'autres organisations de la société civile africaine, pour faire valoir le droit à la libre circulation et affirmer leur désir d'unité. Des opérations sont réalisées aux abords des frontières pour dénoncer les pratiques de racket et les innombrables obstacles à la liberté de circuler qui entravent la route des migrants. S'en dégagent des mots d'ordre communs relatifs au traitement des expulsés mais aussi des migrants à l'échelle internationale. Cette mobilisation transnationale trouve son point d'orgue dans la création d'une assemblée des migrants et l'adoption d'une charte mondiale des migrants 5 à Gorée, en marge du forum social. Celles-ci concrétisent la naissance, par-delà les limites imposées par les frontières nationales, d'un espace de représentation pour les expulsés.

\section{Acteur économique ou politique, victime, témoin, garde-fou...}

Les mobilisations de l'AME depuis 1996 ont permis l'émergence de la figure de l'expulsé comme un acteur politique, une position revendiquée dans une déclaration du 17 décembre 2011 à l'occasion de la célébration de la journée internationale du migrant décrétée par l'ONU et du $15^{\mathrm{e}}$ anniversaire de l'association: «Le migrant n'est pas qu'un acteur économique (transferts financiers), c'est aussi un acteur politique. L'État doit l'aider à faire respecter ses droits dans les pays d'accueil. »

Pourtant, la progressive inscription - renforcée par des partenariats avec des ONG européennes - des associations d'expulsés sur le registre de l'humanitaire a eu tendance à mettre seulement en avant l'image de l'expulsé comme une victime requérant une prise en charge sociale et médicale. La mise en œuvre d'un programme de santé mentale entre 2009 et 2011 par l'AME et Médecins du Monde était emblématique de ces tensions dans les représentations; le primat du registre humanitaire ne risquait-il pas de contribuer à la dépolitisation du mouvement? Par ailleurs, le ralliement de certaines associations, au
Mali, au Cameroun ou au Sénégal, à la stratégie de dissuasion menée par l'UE et l'OIM par l'organisation de campagnes «d'information et de sensibilisation sur les risques de la migration irrégulière » ou par des mesures visant à fixer les expulsés dans leur pays d'origine, fait émerger une autre représentation de l'expulsé, comme témoin et gardefou. Les expulsés sont appelés à témoigner des épreuves qu'ils ont traversées, donnant une authenticité et une crédibilité à cette pédagogie de la dissuasion, dont sont privés les messages portés par les organisations politiques.

La politisation des expulsés est par conséquent un processus complexe. Si la protestation engagée par l'AME à partir des années 1990 semblait inaugurer une critique organisée contre les expulsions à travers le continent africain, la multiplication et la diversification des associations d'expulsés dans différents pays ont abouti à des positionnements politiques variés. De ce mouvement ont surgi des représentations contrastées, faisant passer tour à tour les expulsés d'acteurs de la contestation contre le durcissement des politiques migratoires à des alliés de ces politiques.

\footnotetext{
${ }^{1}$ Archives du Gisti

2 Madiguène Cissé, Parole de sans-papiers, La Dispute, 1999; Ababacar Diop, Dans la peau d'un sans-papiers, Seuil, 1997.

3 L'ARTD, l'AMRK accueillent en 2009 et 2010 les expulsés grâce à des fonds du Cigem et effectuent un suivi des projets de l'OIM pour faciliter la réinsertion des expulsés et leur ancrage durable dans leur pays d'origine.

${ }^{4}$ Grâce à l'émission populaire de la matinée « Bonjour » sur la CRTV (radio télévision publique au Cameroun) et au programme spécial « Magazine Émigration Clandestine ».

${ }^{5}$ Cf. charte-migrants.net/charte-mondiale-desmifgrants/
} 